\title{
¿Qué fue de la "casa común europea"? Percepciones de Europa en la política exterior de Rusia
}

\section{What Happened to the "Common European Home"? Perceptions of Europe in Russia's Foreign Policy}

\author{
Javier Morales Hernández ${ }^{1}$ \\ Universidad Complutense de Madrid (España)
}

Recibido: 16-09-20

Aceptado: 13-10-20

\section{Resumen}

Este trabajo toma como referencia el concepto de "casa común europea" de Gorbachov para estudiar la evolución de las percepciones hacia Europa en la doctrina rusa de política exterior, con el fin de evaluar en qué medida dicho concepto se ha mantenido, transformado o abandonado por completo a lo largo de los mandatos de Yeltsin y Putin. Comenzaremos analizando la formulación original de este argumento en la perestroika, para compararla después con el pensamiento de los dirigentes rusos sobre la identidad europea - o no- de su país. Las principales fuentes primarias empleadas serán las declaraciones de los propios líderes, para comprender cómo estos han definido y articulado discursivamente esta cuestión. Finalmente, en las conclusiones responderemos a estas preguntas iniciales, planteando también sus posibles consecuencias para las relaciones entre Rusia y la UE durante los próximos años.

Palabras-clave: Europa, casa común europea, URSS, Rusia, UE.

\footnotetext{
${ }^{1}$ (jmorales@ucm.es). Profesor Ayudante Doctor de Relaciones Internacionales en la Universidad Complutense de Madrid. Sus principales líneas de investigación son la política exterior de Rusia, la seguridad internacional y la teoría de las Relaciones Internacionales. Entre sus publicaciones más recientes, pueden mencionarse La Unión Europea y Rusia cara a cara: relaciones, conflictos e interdependencias (con Rubén Ruiz-Ramas y Jesús de Andrés, coords., 2020), "Strategic Stability and Great-Power Rivalry in U.S.-Russia Security Relations" (2019) y "Seguridad ontológica y percepciones de amenaza: Rusia ante la ampliación de la OTAN" (2018).

ORCID: https://orcid.org/0000-0001-9909-1077.
} 


\begin{abstract}
This paper takes Gorbachev's concept of "common European home" as a reference to study the evolution of perceptions towards Europe in Russian foreign policy doctrine, in order to assess to what extent this concept has been maintained, transformed or fully abandoned throughout the Yeltsin and Putin presidencies. We will begin by analyzing the original formulation of this argument during perestroika, to later compare it with the Russian leaders' thinking about the European -or non-European- identity of their country. Our main primary sources used will be the statements of the leaders themselves, to understand how they have discursively defined and articulated this issue. Finally, in the conclusions we will answer these initial questions, considering their possible consequences for relations between Russia and the EU in the coming years.
\end{abstract}

Key-words: Europe, Common European Home, USSR, Russia, EU.

\title{
Introducción
}

Las relaciones de Rusia con la mayoría de sus vecinos europeos no han dejado de deteriorarse en los últimos años, especialmente a partir de la revolución en Ucrania en 2014, que parece haber consolidado de forma definitiva el giro prooccidental de este país. A los repetidos enfrentamientos políticos con la OTAN, cuyas sucesivas ampliaciones han sido siempre percibidas por Moscú como una amenaza para su seguridad, se han unido ahora las sanciones de la Unión Europea en respuesta a la anexión ilegal de Crimea, que han acabado con las tímidas iniciativas de cooperación -incluyendo negociaciones para la eliminación de visados- impulsadas en etapas anteriores. Más recientemente, las protestas ciudadanas en Bielorrusia han continuado esta tendencia general hacia el debilitamiento de la influencia rusa en su periferia; a excepción de aquellos territorios, como el este de Ucrania, donde Moscú ha llegado a recurrir a la intervención militar. Este "poder duro" parece ser utilizado por Rusia como alternativa a la ineficacia de su "poder blando"; el cual, pese a sus esfuerzos propagandísticos, se ha demostrado insuficiente para contrarrestar la capacidad de atracción de la UE entre gran parte de los habitantes de nuestro vecindario común.

Más allá de cómo evolucione en el futuro la estrategia exterior de la UE, cabe preguntarse si estas acciones por parte de Rusia representan una ruptura definitiva con su propia identidad europea, sustituyéndola por una reivindicación de su especificidad cultural en oposición a la influencia de Occidente. El discurso cada vez más conservador de Putin en cuestiones 
sociales, como respuesta a los avances en el respeto a la diversidad que se han producido en otros países de Europa, parece encajar con una visión del mundo en términos de "choque de civilizaciones", en la que la globalización liberal sería una amenaza directa para los valores más tradicionales de la sociedad rusa. Por otra parte, el estrechamiento cada vez mayor de las relaciones con China -como vía para salir de su aislamiento internacional tras la anexión de Crimea- puede reforzar a largo plazo la identidad de Rusia como potencia asiática, haciéndole abandonar cualquier sentido de pertenencia a una sociedad internacional europea. Otro indicador de este alejamiento sería la integración económica liderada por Moscú en el espacio eurasiático, que al disminuir la interdependencia entre la economía rusa y las de sus socios comerciales de la UE podría reducir también los incentivos para una reconciliación política entre ambas partes.

El contraste entre el momento presente y otras etapas históricas es especialmente notable si volvemos la vista hacia los años inmediatamente anteriores al desmoronamiento de la URSS. Como es sabido, las reformas internas de Gorbachov tuvieron su correlato en un "nuevo pensamiento" en política exterior, que acabaría poniendo fin al control de Moscú sobre sus países satélites y permitiendo, al mismo tiempo, una conclusión pacífica de la Guerra Fría. Precisamente, la idea de pertenencia a una "casa común europea" fue uno de los principales argumentos empleados por el último líder soviético: Europa dejaba de aparecer como un terreno de conflicto entre bloques para enfatizar, en cambio, las preocupaciones y necesidades compartidas por todos los países del continente. El propio Gorbachov ha seguido defendiendo este concepto hasta hoy, aunque -de forma significativa- sin alinearse por ello con la posición de la UE frente a su país: por el contrario, ha criticado las sanciones en vigor y mostrado incluso su apoyo a la decisión de Putin de anexionarse Crimea (Bonet, 2014; Franchetti, 2016). ¿Cómo pueden ser compatibles estas opiniones con la creencia de que Rusia forma verdaderamente parte de Europa, en un sentido tanto cultural como político? ¿Se trataría de una contradicción intelectual en una persona ya octogenaria o, en cambio, de una comprensión errónea por nuestra parte del pensamiento europeo de Gorbachov, tal como fue articulado originalmente durante la perestroika?

Este trabajo toma como referencia la "casa común europea" para estudiar la evolución de las percepciones hacia Europa en la doctrina rusa de política exterior, con el fin de evaluar en qué medida dicho concepto se ha mantenido, transformado o abandonado por completo a lo largo de los mandatos de Yeltsin y Putin. Comenzaremos analizando la formulación original de este argumento en la etapa de Gorbachov, para compararla después con el pensamiento de los dirigentes rusos sobre la mayor o menor identidad europea de su país. Las principales fuentes primarias empleadas serán las declaraciones de los propios 
líderes - tanto los presidentes como, en el periodo de Yeltsin, los ministros de Asuntos Exteriores ${ }^{2}-$, para comprender cómo estos han definido y articulado discursivamente esta cuestión. Finalmente, en las conclusiones responderemos a estas preguntas iniciales, planteando también sus posibles consecuencias para las relaciones entre Rusia y la UE durante los próximos años.

\section{El concepto de "casa común europea" en la política exterior de Gorbachov}

La formación del pensamiento internacional gorbachoviano se produjo tanto a partir del diálogo con la nueva generación de políticos y académicos de la URSS -que posteriormente serían los arquitectos de las reformas impulsadas por él- como mediante su propio contacto con otros países de Europa, tanto dentro como fuera del bloque socialista. Durante los años setenta, todavía como simple dirigente regional del partido, tuvo la oportunidad poco frecuente entre los ciudadanos soviéticos de visitar Italia, Francia, Alemania, Bélgica y Holanda, tanto en viajes oficiales como turísticos. Esto despertaría en él un creciente interés por los partidos de la izquierda democrática europea, que le llevaría a emplear sus privilegios como miembro del Comité Central para poder acceder a las publicaciones de líderes como Willy Brandt o François Mitterrand. Tras su llegada al poder, mantendría contactos con numerosos políticos e intelectuales europeos: una de sus principales influencias fue precisamente la relación con Felipe González, quien contribuiría a hacer evolucionar su pensamiento en una línea esencialmente socialdemócrata (English, 2000, 182-183, 213).

El concepto de Europa como "casa común" surgió, según el propio Gorbachov, durante sus declaraciones a la prensa en su viaje oficial a París, el primero tras acceder a la secretaría general del PCUS en 1985. No obstante, él mismo ya lo había utilizado el año anterior en el parlamento británico, formando parte de una delegación soviética. Tampoco el término era completamente nuevo: el ministro de Exteriores Andrei Gromyko lo había empleado en 1972, y Leonid Brezhnev lo haría en 1981 (Gorbachev, 1997, 552; Rey, 2004, 34). Sin embargo, fue durante la etapa de Gorbachov cuando se popularizó definitivamente, a raíz de su viaje a Praga en abril de 1987; un año en el que comenzaría a emerger un "vector europeo" de la política exterior soviética,

\footnotetext{
${ }^{2}$ Existe un amplio consenso acerca de la menor influencia en la actual política exterior rusa del ministro de Asuntos Exteriores, cuyo papel ha quedado reducido a implementar las líneas marcadas por Putin y sus colaboradores más cercanos; véase, por ejemplo, el análisis de Galeotti (2016). Por el contrario, la debilidad política y el deficiente estado de salud de Yeltsin permitieron que bajo su mandato los ministros de Exteriores definieran en mayor medida las prioridades a seguir. De ahí que la estrategia de política exterior de los años noventa fuera conocida como "doctrina Kozyrev" y "doctrina Primakov", por el nombre de los responsables de este departamento durante 1991-1996 y 1996-1998, respectivamente.
} 
no subordinado a las relaciones con EE.UU. (Malcolm, 1989, 664). En un discurso dedicado en gran parte a las negociaciones sobre desarme (Associated Press, 1987), y aprovechando el simbolismo de la localización geográfica de Checoslovaquia en el centro del continente, Gorbachov aprovechó para incluir una referencia a su visión de Europa:

Asignamos un significado primordial al rumbo europeo de nuestra política exterior [...]. Estamos decididamente en contra de la división del continente en bloques militares enfrentados, contra la acumulación de arsenales militares en Europa, contra todo lo que es fuente de amenaza de guerra. En el espíritu del nuevo pensamiento introdujimos la idea de la 'casa común europea' [...] [que] significa, sobre todo, el reconocimiento de un cierto todo integral, aunque los Estados en cuestión pertenezcan a diferentes sistemas sociales y sean miembros de bloques político-militares enfrentados entre sí. Este término incluye tanto problemas actuales como posibilidades reales para su solución (Svec, 1988, 990).

Como puede verse, aunque se trataba de una idea definida de forma imprecisa, no se presentaba como una cuestión meramente identitaria o cultural; por el contrario, tenía un carácter eminentemente práctico, directamente vinculado a la situación estratégica. En la visión de Gorbachov, una Europa dividida en bloques militares e inmersa en una carrera de armamentos hacía incrementarse peligrosamente el riesgo de guerra; la "casa común" no supondría per se la desaparición de dichos bloques, pero crearía un sentido de responsabilidad compartida entre las potencias europeas acerca de su obligación de evitar un conflicto armado. Así, el "nuevo pensamiento" se derivaba principalmente del temor a la posibilidad de una guerra nuclear; un escenario que debía evitarse a toda costa, incluso si para ello era necesario situar los valores humanos universales por encima de los intereses de clase (Sodupe y Moure, 2011, 84-85; Casier, 2018, 19).

Naturalmente, en este contexto todavía de Guerra Fría, las relaciones bilaterales entre EE.UU. y la URSS eran un factor determinante en la aproximación de esta última hacia sus vecinos europeos. Aunque Gorbachov niega en sus memorias que el propósito de esa idea de "casa común europea" fuera dividir a los miembros de la OTAN, dejando fuera de él a EE.UU., su relato del diálogo con líderes como Mitterrand deja claro que consideraba a los países de Europa Occidental como potenciales apoyos en las negociaciones con Washington. También critica la visión geopolítica de políticos estadounidenses como Kissinger, a quien acusa de intentar mantener a la URSS aislada del resto del continente (Gorbachev, 1997, 554-558).

La imagen de la "casa común" tuvo inicialmente una función de mera propaganda en la doctrina gorbachoviana, al igual que había ocurrido en la etapa de Brezhnev (Malcolm, 1989, 662; Rey, 2004, 35-37). Se trataba ante todo de 
una campaña de relaciones públicas, destinada a mejorar la imagen del líder soviético ante las sociedades de Europa Occidental, ensalzando la superioridad de la cultura europea -compartida por todos los países del continente, capitalistas o socialistas- respecto de la estadounidense. Además, llamaba a los líderes europeos a estrechar sus relaciones con Moscú como medio de presionar indirectamente a EE.UU. Pero el "nuevo pensamiento" sobre Europa iría mucho más allá de una simple estrategia para dividir a los miembros de la OTAN, dando lugar a una redefinición profunda de la identidad de la URSS como actor internacional; que tendría su plasmación posterior en los acuerdos para la reunificación de Alemania y el abandono de la "doctrina Brezhnev" de soberanía limitada en las relaciones con los demás países socialistas.

Podríamos considerar también este concepto como una modalidad de lo que algunos teóricos de las relaciones internacionales, dentro de la llamada Escuela Inglesa, han denominado "sociedad internacional": un conjunto de Estados que, además de perseguir cada uno sus respectivos intereses nacionales, comparten también algunos intereses y están unidos por ciertas normas e instituciones (Bull, 2005, 65). Por tanto, como afirma Watson $(1992,14)$, el comportamiento de los miembros de la sociedad internacional europea puede explicarse no solamente como una respuesta a las presiones y a los juegos de alianzas basadas en la "razón de estado"; sino a partir de una lógica de "razón de sistema", en la que cada Estado es consciente de que merece la pena hacer funcionar ese modo de convivencia, basado en un mínimo conjunto de reglas del que todos se benefician.

En consecuencia, Gorbachov no pretendía en absoluto afirmar que existiera una comunidad o armonía natural de intereses de todos los países europeos, sino solo un número limitado de problemas y oportunidades compartidos, que suponían un incentivo para la cooperación en áreas concretas. Tampoco planteaba ningún proceso de integración paneuropeo, en el que las soberanías nacionales fueran progresivamente reemplazadas por una entidad supranacional. Como especificó después en su libro Perestroika, la metáfora de la "casa común" no se refería a una familia que comparte una misma vivienda, sino a un bloque de pisos o apartamentos - dom en ruso significa tanto "casa" y "hogar" como "bloque" o "edificio"- en el que residen distintas familias: "la casa es común, es cierto, pero cada familia tiene su propio apartamento, y también hay diferentes entradas". De esta forma:

\footnotetext{
Europa es ciertamente una casa común donde la geografía y la historia han entrelazado los destinos de docenas de países y naciones. Por supuesto, cada una de ellas tiene sus propios problemas y quiere vivir su propia vida, seguir sus propias tradiciones. [...] Pero solo juntos, colectivamente y siguiendo las normas sensatas de la coexistencia, los europeos pueden salvar su casa, protegerla de una conflagración y otras calamidades, hacerla mejor y más segura, y mantenerla en buen funcionamiento (Gorbachev, 1987, 195).
} 
La supervivencia -ante todo, la necesidad de evitar una guerra entre la OTAN y el Pacto de Varsovia- aparecía nuevamente como el principal motivo para una cooperación limitada: esta "comunidad de vecinos" se veía obligada a mantener el "edificio" europeo en condiciones de habitabilidad y seguridad, pero sin interferir en cómo cada familia organizase su vida dentro de su propio domicilio. Aunque la división en alianzas militares fuera definida como una de las causas del riesgo de conflicto, la "casa común europea" no describía un horizonte futuro en el que esas diferencias ya hubieran desaparecido; sino el momento presente, en el que la posibilidad de una guerra obligaba a todos los países del continente a trabajar conjuntamente para evitar que se produjera, a pesar de que cada uno contase con sistemas distintos.

La visión paneuropea de Gorbachov ha sido denominada por Sakwa como "Gran Europa" (Greater Europe), diferenciándola así de la "Europa ampliada" (Wider Europe) identificada con las ampliaciones paralelas de la OTAN y la UE. Esta Europa "incluiría diferentes sistemas sociales [...], pero estaría unida por el respeto a la soberanía y el pluralismo político", sin un único centro ni una misma orientación ideológica (Sakwa, 2020, 110). Así, Europa podría servir en el futuro como ejemplo "de la coexistencia de Estados soberanos y pacíficos, aunque diferentes, que son plenamente conscientes de su interdependencia y fundan sus relaciones mutuas en la confianza"; en cambio, "la muy difundida convicción [...] de que para superar la escisión en Europa hay que "superar el socialismo' [...] es una vía para la confrontación, si no algo peor. A base de estos criterios no habrá unidad europea" (Gorbachev, 1997, 553; Gorbachov, 1990, 35). El líder soviético, como sabemos, no tenía en mente la desaparición del sistema comunista como modelo alternativo, sino su transformación gradual en un socialismo democrático que pudiera coexistir pacíficamente con los países capitalistas y -en última instancia- persuadir a las poblaciones de estos últimos de sus ventajas respecto a la economía de mercado.

Pese a los límites de esta identidad o sentido de pertenencia a una misma sociedad internacional europea, que no eliminaba por completo las diferencias entre sistemas políticos o económicos, tampoco hay que subestimar el avance que supuso respecto a etapas anteriores. Por primera vez, la URSS reconocía abiertamente la existencia de ciertos intereses compartidos con los miembros europeos de la OTAN, sin considerar a estos como simples apéndices o instrumentos del imperialismo estadounidense. En contraste tanto con la etapa zarista como con la soviética, tampoco se recurría a deslegitimar a Europa Occidental como una versión corrupta o degenerada de la "verdadera Europa" liderada por Moscú; sino que se enfatizaban los lazos culturales e históricos entre todas las naciones del continente, miembros de una civilización europea común (Neumann, 2017, 143; Morales Hernández, 2020, 36). 
La metáfora sería desarrollada en sucesivos discursos de Gorbachov durante los años 1988 y 1989. Esta "casa común" tendría cuatro niveles: sus cimientos estarían formados por el Acta Final de Helsinki, especialmente la inalterabilidad de las fronteras europeas. Las plantas superiores se identificaban, de abajo a arriba, con la seguridad colectiva y el desarme, incluyendo la desaparición de los bloques militares; la resolución pacífica de conflictos; la cooperación económica y comercial; $\mathrm{y}$, finalmente, una comunidad cultural (Rey, 2004, 39).

Gorbachov identificaba también cinco "imperativos para una política paneuropea", o áreas donde existían estos problemas compartidos que hacían necesaria una cooperación. En primer lugar, Europa era "un polvorín" de armas tanto nucleares como convencionales. En segundo lugar, incluso una guerra convencional podría llegar a hacer inhabitable el continente, tanto por los avances en la tecnología armamentística como por los efectos de la contaminación en caso de ataque a centrales nucleares o químicas; puede detectarse el impacto en el líder soviético de la catástrofe de Chernóbil, ocurrida el año anterior a la publicación de este libro. En tercer lugar, la alta industrialización de Europa había generado problemas medioambientales que tampoco se veían limitados por las fronteras de cada país. En cuarto lugar, el proceso de integración representado por la Comunidad Económica Europea (CEE) podría agravar la división del continente y, por tanto, las tensiones entre bloques militares, a menos que se establecieran mecanismos de cooperación entre los países socialistas y los miembros de la CEE; pero sin llegar a una integración de todos ellos en una única entidad supranacional. Finalmente, la reducción de las desigualdades Norte-Sur era también una responsabilidad conjunta de todos los europeos (Gorbachev, 1987, 195-196).

La idea de "casa común" no llegaría a convertirse en un proyecto definitivo, como reconoció su propio autor; más allá de una llamada genérica a "dar prioridad a los valores europeos" - sin especificar cuáles serían estos, ni en qué se diferenciarían de los asumidos por otros continentes-y "sustituir el tradicional equilibrio de fuerzas por el equilibrio de intereses". No obstante, la prioridad siguió centrada en todo momento en las cuestiones de seguridad, principalmente las negociaciones sobre desarme (Gorbachov, 1990, 37-38).

\section{La "casa común europea" en la política exterior de Yeltsin}

El modo de actuar de Yeltsin en sus relaciones con otros países se diferenciaba notablemente del de Gorbachov: ante todo, por la carencia de cualquier elaboración ideológica o intelectual significativa en el presidente ruso, cuyo talante personal no era precisamente favorable a la reflexión teórica. Sus 
contactos con los demás líderes europeos estaban marcados por el personalismo, quedando subordinados a la mayor o menor simpatía por su interlocutor. Así, Yeltsin describe en sus memorias sus encuentros informales -"en mangas de camisa"- con Helmut Kohl o Jacques Chirac, a los que llega a calificar de "amigos"; en contraste con Tony Blair, con quien no existía el mismo grado de afinidad, y que "habría estropeado nuestra comodidad psicológica y política" de haber sido invitado a estas reuniones tripartitas, haciéndolas "demasiado oficiales" (Yeltsin, 2001, 147-148).

Sin embargo, a pesar de sus diferencias con Gorbachov, la aproximación del líder ruso no parecía diferenciarse en exceso de la "casa común europea" imaginada por su predecesor; aunque con una presencia menor de las cuestiones militares, en un momento en el que -concluida la Guerra Fría- el peligro de un conflicto nuclear con Occidente ya no estaba en el horizonte. La idea de una "Gran Europa" propuesta por Yeltsin (2001, 149-150) a Kohl y Chirac se basaba en mayor medida en los intercambios económicos y sociales, desde el ámbito educativo hasta las infraestructuras de transporte. No obstante, las preocupaciones de seguridad tampoco habían desaparecido por completo. Esta cooperación trilateral era planteada abiertamente por el presidente ruso como una respuesta a la ampliación de la Alianza Atlántica, intentando crear sin éxito un frente común contra ella:

Todos comprendíamos que nuestra troika estaba llamada a ejercer de contrapeso al desequilibrio producido en Europa tras la aproximación de las fronteras de la OTAN hacia Rusia. [...] Había que hacer algo para oponerse a la presión americana: una voluntad de colaborar, una determinación europea independiente. [...] [Pero] dos años después, estaba claro que afrontábamos la cuestión de la troika de formas distintas. Kohl y Chirac -como garantes de la estabilidad interna europea- querían suavizar la dureza de mi actitud y mis declaraciones sobre la OTAN, mientras que yo solo soñaba con crear un eje Moscú-Berlín-París sobre cuestiones puramente sociales y educativas (Yeltsin, 2001, 149).

La evolución desde un excesivo optimismo y voluntarismo acerca de una cooperación paneuropea estable, característica de los primeros años de Yeltsin, hasta una actitud más desengañada, en la que las cuestiones de seguridad pasaron nuevamente a un primer plano, se vio representada respectivamente por los dos ministros de Exteriores de esta etapa, Andrey Kozyrev y Yevgeni Primakov. El mandato de Kozyrev tuvo como primer objetivo establecer relaciones con los nuevos Estados surgidos de la desintegración de la URSS, así como "apagar fuegos" en las zonas de conflicto de la periferia rusa; pero también emprender una transformación más profunda y ambiciosa de las relaciones con Occidente, superando los límites del "nuevo pensamiento" de Gorbachov. 
Para Kozyrev, la Rusia independiente de principios de los noventa debía adoptar una política exterior completamente nueva, ya que sus intereses eran opuestos a los de la URSS, sin reconocer ninguna continuidad histórica entre las necesidades del país en ambas etapas. Las relaciones internacionales debían quedar subordinadas a las prioridades internas de establecer un sistema democrático y una economía de mercado. Aunque esto debiera realizarse, según él, mediante "contactos libres de ideología con todos los países amantes de la paz, sobre la base de la paridad y la reciprocidad", las prioridades quedaban claras: Rusia debía, ante todo, "encontrar un lugar adecuado como socio y potencial aliado entre las democracias occidentales prósperas", considerando a EE.UU. y al resto de Occidente "no solo como socios, sino como aliados" (Kozyrev, 2019, 123-124, 130).

En esta tarea, el ministro debió enfrentarse tanto a la personalidad impredecible y egocéntrica de Yeltsin como a los sectores nacionalistas dentro y fuera del gobierno, que le consideraban el principal artífice de una "traición" o "cesión" de los intereses rusos frente a Washington. Con la perspectiva de los años transcurridos, parece claro que acabó pagando con su cargo la incompetencia de su presidente, quien acabaría por cesarle a principios de 1996 para no verse perjudicado por las críticas internas a su política exterior. Por otra parte, no hay duda de que efectivamente Kozyrev consideraba la relación bilateral con Washington como el vector más importante para Rusia, y que dedicó a ella sus mayores esfuerzos, pero esto no significa que aceptase ocupar una posición subordinada dentro del orden internacional de la post-Guerra Fría. En una etapa de durísimos ajustes económicos, en la que el apoyo internacional era imprescindible para la supervivencia del país, Moscú carecía de alternativas a corto plazo a la cooperación con EE.UU.; una situación muy distinta a la que se le presentaría después a Putin, con una economía en ascenso gracias a las exportaciones de hidrocarburos.

Si en la "doctrina Kozyrev" la política hacia Europa había quedado subsumida en el marco más amplio de las relaciones con Occidente, con las prioridades más inclinadas hacia el otro lado del Atlántico, esto se mantendría en parte tras el nombramiento de Yevgeni Primakov en la cartera de Exteriores, aunque ahora desde una perspectiva opuesta. El nuevo ministro parecía seguir considerando a Occidente como un bloque relativamente homogéneo bajo liderazgo estadounidense, de forma que las relaciones de Moscú con los aliados europeos de Washington tendrían que ser gestionadas en función de su impacto en las negociaciones con este último. No obstante, el giro respecto a su predecesor consistía en percibir la hegemonía unipolar de EE.UU. como una verdadera amenaza, en lugar de como un aliado y referente democrático a imitar por Rusia en su propia transición interna. Para Primakov, el mandato de Kozyrev habría situado al país en una inmerecida posición de dependencia, 
renunciando incluso a defender los intereses nacionales con tal de ganar la aprobación de Washington:

No todo el mundo en el Ministerio de Asuntos Exteriores, ni mucho menos en otros organismos de política exterior, pensaba que el mundo debía dividirse entre los 'civilizados' y la 'chusma', y que el objetivo más urgente de la nueva Rusia debía ser alcanzar una unión estratégica con los 'civilizados' -nuestros antiguos adversarios de la Guerra Fría- a cualquier precio. Al mismo tiempo, el formato previsto situaba a Rusia como la seguidora y a Occidente como el líder (Primakov, 2004, 126).

Esta reorientación de la acción exterior -claramente identificada con los postulados del realismo político- tampoco estaba exenta de pragmatismo: Rusia "podía y debía buscar activamente una asociación equitativa con todos", trabajando con otros países en áreas donde los respectivos intereses fueran coincidentes. Y cuando esto no sucediera, "debemos intentar encontrar soluciones que no sacrifiquen los intereses nacionales vitales de Rusia, ni lleven a la confrontación" (Primakov, 2004, 126-127). Por otra parte, la preocupación por la supervivencia ya no era tan acuciante como en el periodo de Gorbachov, aunque las cuestiones de seguridad siguieran siendo relevantes. La principal amenaza sería ahora la ampliación de la OTAN, no solo en un sentido estrictamente militar -de hecho, Moscú no consideraba que la Alianza pretendiera lanzar un ataque contra ella- sino más bien político: impedir a Rusia ejercer como un actor influyente y un polo de poder dentro del nuevo sistema multipolar (Primakov, 2005, 208-209).

El debate sobre la política exterior rusa de la década de los noventa destaca, por tanto, por la ausencia de una identidad específicamente europea: mientras que liberales como Kozyrev consideraban que Rusia debía ser parte de Occidente, tomando a EE.UU. como modelo en mayor medida que a las democracias europeas, nacionalistas como Primakov defendían la autonomía de su país como una potencia por derecho propio, que mantuviese una presencia en distintas regiones pero sin alinearse o identificarse exclusivamente con ninguna de ellas. Por ejemplo, es significativo que ninguna de las cinco posibles autoidentificaciones estudiadas por Clunan $(2009,60)$-occidental, estatista, nacional-restauracionista, neocomunista y eslavófila- se definiera específicamente por un sentido de pertenencia a Europa. Sin embargo, otros autores han detectado algunas similitudes entre la multipolaridad defendida por Primakov y la "casa común" de Gorbachov; la cual, en el fondo, consistía también en un mismo continente con varios centros de poder, cada uno de ellos con sistemas políticos y económicos diversos, aunque unidos por la preocupación ante ciertos problemas comunes (Casier, 2018, 23). 


\section{La "casa común europea” en la política exterior de Putin}

El comienzo de la presidencia de Putin, tras la renuncia de Yeltsin a finales de 1999, se caracterizó por unas relaciones relativamente positivas con los países occidentales. Sus primeras declaraciones enfatizaron repetidamente la identidad europea de Rusia, pareciendo moderar en cierta medida la influencia del realismo político en los años inmediatamente anteriores, que se había agudizado por el enfrentamiento con la OTAN sobre la guerra de Kosovo. Así, el nuevo líder llegó a afirmar que toda Rusia-incluyendo sus regiones asiáticasera "parte de la cultura europea occidental", "un país europeo en términos de su situación geográfica, su cultura y su nivel de integración económica" (Gevorkian, Kolesnikov y Timakova, 2000, 155-156; Smith, 2002, 2).

No obstante, el supuesto "eurocentrismo" de Putin no se correspondía con la importancia concedida al espacio postsoviético, ni su rechazo a la unipolaridad del sistema internacional de la post-Guerra Fría, que se deseaba sustituir por un sistema multipolar en el que existieran varios polos alternativos a la hegemonía estadounidense (Lo, 2003, 17, 102-105). Sus referencias iniciales a la identidad europea de Rusia debían interpretarse, por tanto, de forma mucho más limitada:

\footnotetext{
Putin puede ser europeo por afinidad emocional e intelectual -aunque esto es debatible-, pero la cultura estratégica en la que creció siempre ha tomado a EE.UU. como su referencia básica. Además, Washington se mantiene como el actor dominante en las áreas de interés fundamental para Moscú: la lucha contra el terrorismo internacional, la integración en la economía global y la agenda de desarme estratégico (Lo, 2003, 7).
}

Esta búsqueda de un papel relevante explica que se mantuvieran durante un tiempo las reuniones trilaterales entre Rusia, Francia y Alemania, que escenificaban el alineamiento de Moscú con la "vieja Europa" -en la expresión de Donald Rumsfeld- frente a las políticas de la Administración Bush, especialmente en cuanto a la invasión de Irak. Una vez desaparecidos los motivos coyunturales de esa acción conjunta, los incentivos para profundizar en la cooperación UE-Rusia han ido disminuyendo para ambas partes de forma sostenida..

Putin mantendría así la concepción multivectorial heredada de la etapa anterior, según la cual Rusia debía mantener una política exterior activa hacia distintas regiones simultáneamente, evitando que la prioridad concedida a una de ellas -como había ocurrido durante el mandato de Kozyrev con las relaciones con EE.UU.- se tradujese en un abandono de las demás. Por ejemplo, el Concepto de Política Exterior aprobado en 2000 situaba como primera prioridad geográfica a la Comunidad de Estados Independientes (CEI); mientras que Europa aparecía en una segunda posición, destacando tanto los 
intereses rusos en relación con la OSCE, la UE y la OTAN como la importancia de la cooperación bilateral con otras potencias europeas, como el Reino Unido, Alemania, Italia y Francia (Federation of American Scientists, 2000). Sin embargo, en torno a 2004-2007, el "eurocentrismo" inicial de Putin sería reemplazado por un mayor distanciamiento respecto de la UE, sin pretender establecer una cooperación tan estrecha. A partir de entonces, las relaciones ruso-europeas se basarían exclusivamente en intereses, no en una "comunidad de valores compartidos" (Casier, 2018, 24).

La evolución interna de Rusia en una dirección más autoritaria ha tenido también su eco en la política exterior: a diferencia de lo que sucedía durante la perestroika, la aproximación hacia Europa ya no se fundamenta en la universalidad de los derechos y libertades fundamentales, como sucedía en la visión de Gorbachov. En los últimos años, el discurso putiniano sobre la identidad de Rusia y su grado de pertenencia a Europa ha destacado cada vez más las diferencias de valores, contraponiendo el mayor conservadurismo de la sociedad rusa a la "degradación moral" de los países occidentales. Esto ha permitido a Putin intentar presentarse de nuevo como líder de una "verdadera Europa", donde los valores tradicionales supuestamente abandonados por Occidente aún resistirían a las presiones de la globalización liberal:

Vemos cómo muchos de los países euroatlánticos están, de hecho, rechazando sus raíces, incluyendo los valores cristianos que constituyen la base de la civilización occidental. Niegan los principios morales y todas las identidades tradicionales [...] equiparan a las familias numerosas con las parejas del mismo sexo, y la creencia en Dios con la creencia en Satán. [...] En muchos países europeos, la gente tiene vergüenza o miedo de hablar de sus creencias religiosas (Johnson's Russia List, 2013).

El Concepto de Política Exterior de 2016 se corresponde con este distanciamiento, aunque manteniendo -afortunadamente- un lenguaje más diplomático que los excesos retóricos del presidente ruso. El espacio postsoviético se mantiene como la primera prioridad geográfica, reforzada por el proceso de integración económica en Eurasia. En cambio, la UE aparece ahora unida a la OTAN, describiendo ambas ampliaciones como dos caras de una misma "expansión geopolítica" occidental, a la que se responsabiliza de la crisis en las relaciones mutuas. No obstante, se destaca también que Rusia sigue considerando a la UE como un importante socio comercial, y que no se descarta la cooperación en aquellas otras áreas donde sea posible (Ministry of Foreign Affairs of the Russian Federation, 2016). 


\section{Conclusiones}

Como hemos visto, la visión paneuropea de la "casa común" no ha desaparecido por completo de la política exterior de Moscú: varios de sus elementos han seguido presentes en el pensamiento internacional de los líderes rusos, aunque con algunas transformaciones de su significado primigenio. La principal causa de estas diferencias es el contexto internacional en el que han debido aplicarse.

Paradójicamente, el peligro real de un conflicto armado entre bloques durante la Guerra Fría fue lo que incentivó a Gorbachov a buscar un mayor entendimiento con los demás países de Europa, considerando que no había alternativas al diálogo si se quería garantizar la supervivencia del continente. En cambio, en las etapas más recientes ya no se han percibido las relaciones rusoeuropeas como una cuestión de "vida o muerte", sino basada exclusivamente en la conveniencia y los intereses; lo que ha llevado al Kremlin a emplear estrategias más basadas en la coerción y la confrontación, sin temor a una escalada que pudiera derivar en una guerra nuclear.

Este cálculo, sin embargo, se ha demostrado arriesgado en escenarios como Ucrania, donde Rusia ha actuado de una forma crecientemente irreflexiva e imprudente, sin prever las consecuencias negativas a largo plazo de sus acciones para sus propios intereses y reputación internacional. Mientras que Gorbachov tuvo como prioridad la reducción de las tensiones militares, a través del desarme y la "suficiencia defensiva", Putin ha adoptado una actitud cada vez más agresiva y provocativa, llegando a tomar decisiones -como la ocupación y anexión de Crimea-que habrían sido impensables en la perestroika, precisamente por el temor a que desencadenasen un enfrentamiento armado imposible de controlar.

Por otra parte, el "giro conservador" en Rusia durante los últimos años hace todavía más difícil que se vuelva a una cooperación pragmática. El maximalismo del rechazo de Putin hacia las sociedades más diversas -una actitud que, recordemos, tampoco es exclusiva de su país, sino compartida por los sectores más intolerantes de los propios miembros de la UE- contrastan con la flexibilidad del planteamiento gorbachoviano, basado en la coexistencia pacífica entre países con distintos modelos de convivencia, libremente decididos por cada uno de ellos. 


\section{Referencias bibliográficas:}

Associated Press (1987): "Excerpts of Gorbachev's speech in Prague", $A P$ News, 11/04/1987, <https://apnews.com/16ec430b83976acc03c1c194b2 $\mathrm{d} 3 \mathrm{fc} 42>$.

Bonet, Pilar (2014): "Es urgente construir la casa común europea", El Pais, $\quad$ 07/12/2014, <https://elpais.com/internacional/2014/12/05/ actualidad/1417795313_638607.html>.

Bull, Hedley (2005): La sociedad anárquica. Un estudio sobre el orden en la política mundial, Madrid, Los Libros de la Catarata.

Casier, Tom (2018): "Gorbachev's «Common European Home» and its relevance for Russian foreign policy today", Debater a Europa, 18, pp. 17-34, <https:// doi.org/10.14195/1647-6336_18_2>.

Clunan, Anne L. (2009): The social construction of Russia's resurgence: aspirations, identity, and security interests, Baltimore, Johns Hopkins University Press.

English, Robert D. (2000): Russia and the idea of the West: Gorbachev, intellectuals, and the end of the Cold War, Nueva York, Columbia University Press.

Federation of American Scientists (2000): The Foreign Policy Concept of the Russian Federation, <https://fas.org/nuke/guide/russia/doctrine/econcept. htm>.

Franchetti, Mark (2016): "Gorbachev says Putin was right to snatch Crimea", The Sunday Times, 22/05/2016, <https://www.thetimes.co.uk/article/gorbachevsays-putin-was-right-to-snatch-crimea-3g2wjr0fc $>$.

Galeotti, Mark (2016): "Free Sergei Lavrov!", Foreign Policy, <https:// foreignpolicy.com/2016/02/17/free-sergei-lavrov-putin-russia-syria/>.

Gevorkian, Natalia, Andrei Kolesnikov y Natalia Timakova (2000): Ot pervogo litsa. Razgovori s Vladimirom Putinim, Moscú, Vagrius.

Gorbachev, Mikhail (1997): Memoirs, Londres, Bantam.

Gorbachev, Mikhail (1987): Perestroika: new thinking for our country and the world, Nueva York, Harper \& Row.

Gorbachov, Mijaíl (1990): “La unificación europea debe ser una tarea común”, en Hacia la casa común europea. Una nueva política para Europa y el mundo, Barcelona, Círculo de Lectores, pp. 31-50.

Johnson's Russia List (2013): "Transcript: [Putin at] Meeting of the Valdai International Discussion Club [partial transcript]", Johnson's Russia List, $<$ https://russialist.org/transcript-putin-at-meeting-of-the-valdai-internationaldiscussion-club-partial-transcript/>.

Kozyrev, Andrei (2019): The firebird: the elusive fate of Russian democracy. A memoir, Pittsburgh, University of Pittsburgh Press. 
Lo, Bobo (2003): Vladimir Putin and the evolution of Russian foreign policy, Oxford, Blackwell.

Malcolm, Neil (1989): "The «Common European Home» and Soviet European Policy”, International Affairs, 65, 4, pp. 659-676, <https://doi. org/10.2307/2622579>.

Ministry of Foreign Affairs of the Russian Federation (2016): Foreign Policy Concept of the Russian Federation, <https://www.mid.ru/en/foreign_policy/ official_documents/-/asset_publisher/CptICkB6BZ29/content/id/2542248>.

Morales Hernández, Javier (2020): "Identidades e intereses de Rusia hacia Europa: de la inclusión limitada a la (auto-)exclusión", en Rubén RuizRamas, Jesús De Andrés Sanz y Javier Morales Hernández (eds.), La Unión Europea y Rusia cara a cara: relaciones, conflictos e interdependencias, Valencia, Tirant lo Blanch, pp. 27-60.

Neumann, Iver B. (2017): Russia and the idea of Europe: a study in identity and international relations, $2^{\text {a }}$ ed. Abingdon, Routledge.

Primakov, Yevgeny (2005): "International relations on the eve of the 21st century: problems and prospects", en Andrei Melville y Tatiana Shakleina (eds.), Russian foreign policy in transition: concepts and realities, Budapest, Central European University Press, pp. 207-219.

Primakov, Yevgeny (2004): Russian crossroads: toward the new millennium, New Haven, Yale University Press.

Rey, Marie-Pierre (2004): “«Europe is our common home»: a study of Gorbachev's diplomatic concept”, Cold War History, 4, 2, pp. 33-65, <https://doi.org/10.1 080/14682740412331391805>.

Sakwa, Richard (2020): "Rusia, Ucrania y la UE entre dos modelos: monismo frente a pluralismo", en Rubén Ruiz-Ramas, Jesús De Andrés Sanz y Javier Morales Hernández (eds.), La Unión Europea y Rusia cara a cara: relaciones, conflictos e interdependencias, Valencia, Tirant lo Blanch, pp. 107-122.

Smith, Mark A. (2002): Russia and the West, Conflict Studies Research Centre, Camberley, Royal Military Academy Sandhurst.

Sodupe, Kepa, y Leire Moure (2011): “Conceptos soviéticos fundamentales de las Relaciones Internacionales”, en Kepa Sodupe y Leire Moure (eds.), Rusia en la era postsoviética, Bilbao, Servicio Editorial de la Universidad del País Vasco, pp. 61-103.

Svec, Milan (1988): “The Prague Spring: 20 years later", Foreign Affairs, 66, 5, pp. 981-1001, <https://doi.org/10.2307/20043574>.

Watson, Adam (1992): The evolution of international society, Londres, Routledge. Yeltsin, Boris (2001): Midnight diaries, Londres, Phoenix. 